\title{
Um novo extrator de características para aplicações embarcadas de detecção de falhas em motores elétricos
}

\author{
Lucas C. da Silva ${ }^{1}$, Elias T. Silva $\mathrm{Jr}^{2}$, Isabel F. Costa ${ }^{3}$ \\ Computer Science Department \\ Federal Institute of Education, Science and Technology of Ceara - IFCE \\ Fortaleza, Brasil \\ lucas.costa@lit.ifce.edu.br ${ }^{1}$ \\ elias@ifce.edu.br ${ }^{2}$,isabel@lit.ifce.edu.br ${ }^{3}$
}

\begin{abstract}
Os motores de indução são utilizados em diversas aplicações industriais devido à sua alta eficiência. Além disso, o custo por interrupção de operação não plenejada dessas máquinas costuma ser elevado. Por essa razão, há um interesse da indústria e da comunidade científica em desenvolver soluções para detectar antecipadamente falhas nesses motores. Muitos métodos de detecção de padrões têm sido propostos a partir de diferentes sinais extraídos do motor e usando diferentes combinações de extratores de características com algoritmos de classificação; alguns com elevado sucesso, como a combinação GLCM (GrayLevel Co-occurrence Matrix) e SVM (Support vector machine). Este trabalho propõe uma abordagem nova para utilização do GLCM em sinais no tempo, mas evitando a criação de uma imagem intermediária para representação do sinal de entrada. A técnica proposta é avaliada em estação de trabalho e em plataforma embarcada, com resultados de taxa de acerto, tempo de computação e uso de memória.
\end{abstract}

Palavras-chave-GLCM, Motores de indução, aprendizado de maquina, Sistemas embarcados, extrator de caracteristica.

\section{INTRODUÇÃO}

Os motores de indução (MI) são utilizados em diversas aplicações industriais para conversão de energia elétrica em mecânica, devido a sua alta eficiência, robustez, confiabilidade e baixo custo de manutenção. Os motores de indução apresentam falhas, devido a vários motivos como envelhecimento, condições de instalação, aplicações inadequadas, falta de manutenção preventiva e esforços mecânicos ou elétricos [1]. Dentre as falhas mais comuns, conforme lista em [2], está o curto-circuito entre as espiras do enrolamento, que é a segunda mais importante.

Nesse cenário, há uma tendência da indústria e da comunidade de pesquisa em buscar soluções para detectar falhas em motores de indução conforme indicado em revisões como [2] e [3]. Além disso, com o impacto dos conversores de frequência, surge a possibilidade de diagnósticos preventivos por técnicas não invasivas através de monitoramento online constante. Algumas pesquisas indicam que esse diagnóstico pode ser feito usando algoritmos de inteligência artificial, conforme visto em trabalhos como [4], [5].
Uma forma de uso de algoritmos de aprendizagem de máquina usando sinais como entrada que vem sendo explorada recentemente, é a utilização de uma transformação do sinal em imagem, como mostrado no trabalho [6] que utiliza diferentes técnicas para este fim. Com a imagem gerada é realizada uma análise na textura da imagem, visando a extração de caracteristicas usando GLCM. Embora apresente bons resultados em taxas de acerto, esta transformação do sinal em uma imagem pode causar um gasto de recursos para armazenamento da imagem gerada.

Nesse contexto, este trabalho descreve uma abordagem para utilização do extrator de caracteristicas GLCM em sinais no tempo, de forma que não haja a transformação do sinal de entrada, tanto em imagens como em outros domínios.

\section{TÉCNICA PROPOSTA SLCM (SIGNAL LEVEL CO-OCURRENCE MATRIX).}

As técnicas de reconhecimento de padrões em sinais tipicamente utilizam um modelo onde o sinal passa por algum tipo de extrator de características, antes de ser entregue ao algoritmo classificador. Um extrator de características muito utilizado na literatura é o GLCM [7] onde, dada uma imagem 2D, é gerada uma matriz de co-ocorrência e desta matriz são extraidos atributos estatísticos que evidenciam a mudança de textura na imagem.

A técnica aqui proposta visa à extração de características de sinais no dominio do tempo, gerando uma matriz de coocorrência. Para isso, o sinal no tempo passa pelas seguintes etapas:

1) Digitalização: Conversão analógico para digital, geralmente entregue em 12 bits pelo ADC.

2) Discretização: Consiste em diminuir a quantidade de bits usada para representar os valores. Os números digitalizados em 12 bits são convertidos conforme alguma potência de 2, levando a uma redução no tamanho da matriz de co-ocorrencia.

3) Criação da Matriz de co-ocorrência: A estrutura final dessa matriz é a mesma do GLCM [7], mas o método de geração é diferente, já que a entrada será um vetor de 1D. Na expressão 1 , um sinal $S$, dá origem 
a uma matriz de co-ocorrência $C M$, que é inicializada com zeros. Em um determinado passo $t$, se incrementa o valor do elemento de $C M$ localizado na linha de valor $S(t)$ e coluna $S(t+1)$. Para um exemplo de um sinal discretizado em 8 bits, a matriz terá 256 × 256 elementos. A figura 1 mostra um exemplo de geração de uma matriz de co-ocorrência de 2 bits.

4) Cálculo de estatísticas: Um conjunto de estatísticas para se aplicar sobre a matriz de co-ocorrência é apresentado e descrito em [7]. Outras expressões foram adicionadas em [8] e [9] chegando a um total de 24. Esses valores calculados são usados como características, entregues ao algoritmo de classificação.

$$
C M[S(t), S(t+1)]=C M[S(t), S(t+1)]+1
$$

O motor elétrico sob observação foi instrumentado com um sensor de fluxo magnético. O sinal obtido é digitalizado por um microcontrolador, encarregado de executar os algorítimos de extração de características (SLCM) e de classificação.

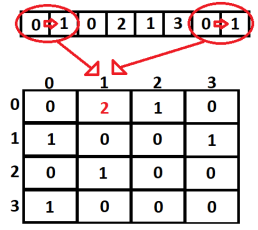

Fig. 1: Exemplo de geração de uma matriz de co-ocorrência de 2 bits.

\section{CONJUNTO DE DADOS}

Esta seção apresenta o conjunto de dados usado para avaliar o extrator de características. Trata-se de uma aplicação de detecção de falhas em motores de indução trifásicos, que utiliza sinais de fluxo magnético extraídos em diferentes condiçoes de operação, dividas em 7 classes. As classes foram dispostas por ordem de severidade, onde a classe 1 representa sem falha e classe 7 a falha mais grave. Cada classe é composta por cerca de 350 amostras e cada amostra possui um sinal com 100.000 pontos [10]. Os mesmos sinais de fluxo magnético são usados pra gerar os diversos experimentos. As seções seguintes mostram como cada um deles é gerado.

\section{TÉCNICAS SIMILARES}

O método de extração de atributos aqui proposto, o SLCM, produz uma matriz de coocorrência, a exemplo do GLCM [7][9], que é comumente usado para extrair atributos de imagens. Por essa razão, outros métodos que trazem técnicas de extração usadas em imagens (duas dimensões) para aplicações que usam sinais de uma dimensão são usadas para comparação.

A figura 2 apresenta um esboço dos experimentos realizados neste trabalho. No conjunto de dados obtido, uma exploração é feita buscando diminuir o tamanho das amostras sem comprometer a taxa de acerto do classificador. Essa avaliação é feita na seção V. Técnicas de geração de atributos que podem ser comparadas com a técnica proposta são STFT [6], Geração de gráfico no tempo [11] e geração de imagem chão-teto. Devido ao custo computacional elevado, somente a técnica de geração de imagem chão-teto será utilizada na plataforma embarcada.

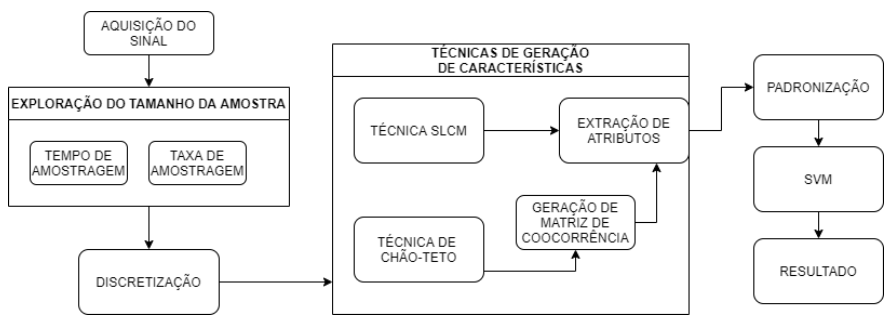

Fig. 2: Experimentos de exploração realizados.

\section{A. Geração de imagem chão-teto.}

Esta técnica consiste na geração de uma imagem 2D em escala de cinza através da conversão de um sinal $1 \mathrm{D}$, podendo servir de entrada para um GLCM [12], [13].

Para melhor comparação, o sinal é discretizado, a exemplo do que ocorre com o SLCM.

Um sinal $S$ com $N$ pontos no tempo permite gerar uma imagem de dimensões $\mathrm{M}$ x M. Para $\mathrm{M}=\lceil\sqrt{N}\rceil$, a coordenada do pixel correspondente ao i-ésimo valor da amostra é dada pela expressão:

$$
p(j, k)=S(i) \mid j=\left\lfloor\frac{i}{M}\right\rfloor, k=\left\|\frac{i}{M}\right\|
$$

Desta forma, para cada valor do sinal $S$ existe um Pixel na imagem que representa este valor. Com isso a imagem gerada é equivalente a uma imagem em escala de cinza, servindo então como entrada para qualquer extrator de caracteristicas de imagens.

Neste trabalho, esta técnica será utilizada em conjunto com o extrator de caracteristicas GLCM.

\section{EXPLORAÇÃO DO ESPAÇO DE PROJETO - REDUÇÃO NO TAMANHO DA AMOSTRA.}

Com o objetivo de reduzir o tamanho da amostra sem perda significativa de qualidade do sinal na etapa de classificação, algumas técnicas para redução da amostra foram aplicadas. Posteriormente seus resultados serão analisados e evidenciados os ganhos de cada técnica.

Nesse procedimento são criados novos conjuntos de dados, reduzindo taxa de amostragem e tamanho da amostra. Para cada conjunto novo de amostras, se repete o processo de treinamento do classificador. Isso permite avaliar a influência das reduções nas taxas de acerto do sistema extrator-classficador. Ao ser aplicada em diferentes métodos de extração de características, é possivel avaliar o quanto cada método é imune a essas reduções. Do ponto de vista do classificador, essas reduções podem significar a também a redução nas informações (caracterísicas) que lhe permitem distinguir melhor entre as classes do problema. 


\section{A. Redução na taxa de amostragem}

Esta exploração visa a avaliar os impactos da redução da frequencia de amostragem nas taxas de acerto do sistema extratorclassificador. Os beneficios de reduzir a taxa de amostragem são: (1) menor uso de memória para armazenamento do vetor de pontos obtido do sensor, e o (2) uso de um conversor analógico-digital de menor custo.

$\mathrm{O}$ processo ocorre com a geração de amostras em frequencias de amostragem cada vez menores, respeitando o teorema de Nyquist, reduzindo a quantidade de pontos. O sinal original foi amostrado com uma $F s$ de $10 \mathrm{kHz}$. na primeira rodada, as frequências acima de $2,5 \mathrm{kHz}$ são filtradas e $F s$ assume o valor de $5 \mathrm{Khz}$. Com isso a quantidade de pontos é reduzida à metade totalizando 50.000 pontos. Em seguida o processo é repetido para obter um segundo corte.

$$
F c=\frac{F s}{2}-(F s \times 0.06)
$$

Foram gerados ao todo seis novos conjuntos de amostras, com frequencias de amostragem de $5 \mathrm{kHz}, 2,5 \mathrm{kHz}, 1,25 \mathrm{kHz}$, $625 \mathrm{~Hz}, 312 \mathrm{~Hz}, 156 \mathrm{~Hz}$, nomeados de $M 1$ a $M 6$ respectivamente. Juntamente com o conjunto com frequência de amostragem original de $10 \mathrm{kHz}$, totalizam sete conjuntos de amostras. Na Tabela ??, Fs é a frequencia de amostragem e $N$ é o número de pontos de cada amostra do conjunto resultante.

\section{B. Redução no tempo de amostragem}

Esta exploração visa a avaliar os impactos da redução no tamanho da amostra nas taxas de acerto do sistema extratorclassificador. Os benefícios de reduzir o tamanho da amostra são: (1) menor uso de memória para armazenamento do vetor de pontos obtido do sensor, e (2) menor tempo de obtenção da amostra, que leva à possibilidade de emitir maior quantidade de diagnósticos por unidade de tempo, ou ainda à possibildiade de economizar energia, desligando o sistema por mais tempo de ociosidade.

Esta técnica realiza tão somente um corte no sinal no tamanho desejado, descartando o restante dos pontos do sinal. Para o processo de exploração, a cada corte o sinal é dividido ao meio e somente a primeira metade do sinal é tomada. Esta técnica permitiria um aumento no número de amostras para o treinamento do classificador. Entretanto, os dados excendentes não serão usados a fim de mantar a comparação justa entre os diferentes tamanhos de amostra.

Os 7 conjuntos resultantes, possuem cada um amostras de tamanho variando de 50.000 a 1.562 pontos, reduzindo em potencias de 2 , além do original, que possui amostras de 100.000 pontos.

\section{AVALIAÇÃO DOS EXTRATORES DE CARACTERÍSTICAS}

Para todas as técnicas mostradas nas seções a seguir, o extrator de características está baseado na matriz de co-ocorrência de [7] e no conjunto de 24 atributos estatísticos [8] [9]. Todos os experimentos utilizam como classificador um SVM [14] com kernel RBF. O SVM é utilizado por ser um algoritmo estado da arte, com bons resultados em aplicações similares e por ser viável sua implantação em microcontroladores.
Para avaliação das taxas de acerto, o conjunto de dados foi dividido tomando $80 \%$ das amostras de cada classe para treinamento e $20 \%$ para testes. Para ajuste dos parâmetros do SVM é usada uma busca em grade com validação cruzada $(k=2)$.

Após este processo, um modelo é gerado e validado usando o conjunto de testes com os parametros encontrados, sendo este processo corresponde a uma rodada do experimento. Posteriormente, o conjunto é aleatoriamente dividido em treino e teste para uma nova rodada. Ao todo foram executadas 50 rodadas em cada técnica investigada e os resultados refletem a média dessas 50 rodadas.

\section{A. Experimento usando a técnica SLCM}

Neste experimento é aplicada a técnica de SLCM para geração da matriz de co-ocorrência do sinal. Esta matriz é utilizada para o processo de geração das características, sem ser necessária a conversão do sinal em uma imagem $2 \mathrm{D}$.

Ao gerar os 24 atributos do GLCM, os valores passam por um processo de padronização de cada amostra, que consiste em subtrair o valor da média e dividir pelo valor do desvio padrão.

\begin{tabular}{|l|l|l|l|l|l|l|l|}
\hline & NORMAL & HI-1 & HI-2 & HI-3 & LI-1 & LI-2 & LI-3 \\
\hline NORMAL & 96.8 & 0.4 & 1.9 & 0.3 & 0.6 & 0.0 & 0.0 \\
\hline HI-1 & 1.6 & 85.7 & 0.0 & 0.0 & 12.4 & 0.3 & 0.0 \\
\hline HI-2 & 1.9 & 0.4 & 95.3 & 1.2 & 0.8 & 0.4 & 0.0 \\
\hline HI-3 & 0.2 & 0.1 & 1.1 & 98.6 & 0.1 & 0.0 & 0.0 \\
\hline LI-1 & 0.1 & 10.8 & 0.1 & 0.3 & 88.7 & 0.0 & 0.0 \\
\hline LI-2 & 0.1 & 0.1 & 0.2 & 0.0 & 0.0 & 99.4 & 0.3 \\
\hline LI-3 & 0.0 & 0.0 & 0.0 & 0.0 & 0.0 & 0.3 & 99.7 \\
\hline
\end{tabular}

TABELA I: Matriz de confusão ao usar a técnica SLCM (seção II) e todos os 24 atributos GLCM.

A Tabela I mostra a matriz de confusão dos resultados obtidos neste experimento usando todos os 24 atributos disponiveis. Em [15] é apresentado um conjunto de 10 atributos escolhidos com base nos gastos de memória e tempo, com resultados de acurácia similares ao uso de todos os 24 atributos e uma redução significativa nos recursos utilizados. Aplicando estes atributos a este problema a matriz confusão resultante pode ser vista na tabela II.

\begin{tabular}{|l|l|l|l|l|l|l|l|}
\hline & NORMAL & HI-1 & HI-2 & HI-3 & LI-1 & LI-2 & LI-3 \\
\hline NORMAL & 95.6 & 1.4 & 2.0 & 0.5 & 0.2 & 0.0 & 0.3 \\
\hline HI-1 & 2.3 & 76.5 & 0.5 & 0.5 & 20.3 & 0.0 & 0.0 \\
\hline HI-2 & 2.9 & 0.8 & 94.0 & 1.4 & 0.2 & 0.8 & 0.0 \\
\hline HI-3 & 0.9 & 0.4 & 1.1 & 97.2 & 0.2 & 0.2 & 0.0 \\
\hline LI-1 & 0.4 & 23.3 & 0.4 & 0.4 & 75.5 & 0.0 & 0.0 \\
\hline LI-2 & 0.1 & 0.5 & 0.9 & 0.1 & 0.2 & 98.2 & 0.0 \\
\hline LI-3 & 0.0 & 0.0 & 0.5 & 0.0 & 0.0 & 0.4 & 99.1 \\
\hline
\end{tabular}

TABELA II: Matriz de confusão ao usar a técnica SLCM (seçao II) e 10 atributos GLCM.

\section{B. Experimento usando a técnica de chão-teto}

Neste experimento é aplicada a técnica de chão-teto (seção IV-A). A imagem gerada passa pelo processo completo do extrator de caracteristicas GLCM. Para fins de comparação com 
outras técnicas, os parametros para GLCM são: vizinhança 1 e $\theta=0^{\circ}$. Os 24 atributos após extraídos passam pelo mesmo processo de padronização.

\begin{tabular}{|l|l|l|l|l|l|l|l|}
\hline & NORMAL & HI-1 & HI-2 & HI-3 & LI-1 & LI-2 & LI-3 \\
\hline NORMAL & 97.1 & 0.3 & 2.1 & 0.2 & 0.3 & 0.0 & 0.0 \\
\hline HI-1 & 0.5 & 90.4 & 0.6 & 0.1 & 8.1 & 0.2 & 0.0 \\
\hline HI-2 & 2.2 & 0.6 & 94.6 & 1.4 & 0.8 & 0.4 & 0.0 \\
\hline HI-3 & 1.1 & 0.0 & 0.8 & 98.1 & 0.0 & 0.0 & 0.0 \\
\hline LI-1 & 0.3 & 9.9 & 0.2 & 0.5 & 89.0 & 0.0 & 0.0 \\
\hline LI-2 & 0.0 & 0.0 & 0.4 & 0.0 & 0.0 & 99.5 & 0.1 \\
\hline LI-3 & 0.0 & 0.0 & 0.0 & 0.0. & 0.0 & 0.5 & 99.5 \\
\hline
\end{tabular}

TABELA III: Matriz de confusão ao usar a técnica de chão-teto (seção IV-A) e todos os 24 atributos GLCM.

A Tabela III mostra a matriz de confusão dos resultados obtidos neste experimento usando todos os 24 atributos disponiveis. Já a Tabela IV mostra os resultados obtidos usando os atributos sugeridos em [15].

\begin{tabular}{|l|l|l|l|l|l|l|l|}
\hline & NORMAL & HI-1 & HI-2 & HI-3 & LI-1 & LI-2 & LI-3 \\
\hline NORMAL & 95.9 & 0.5 & 2.6 & 0.7 & 0.1 & 0.3 & 0.0 \\
\hline HI-1 & 1.4 & 78.3 & 0.9 & 0.0 & 19.4 & 0.0 & 0.0 \\
\hline HI-2 & 3.6 & 0.7 & 92.5 & 1.9 & 0.6 & 0.7 & 0.0 \\
\hline HI-3 & 1.2 & 0.1 & 1.2 & 97.5 & 0.0 & 0.1 & 0.0 \\
\hline LI-1 & 0.7 & 23.3 & 0.7 & 0.1 & 75.1 & 0.0 & 0.0 \\
\hline LI-2 & 0.1 & 0.1 & 1.1 & 0.2 & 0.0 & 98.6 & 0.0 \\
\hline LI-3 & 0.0 & 0.0 & 0.5 & 0.0. & 0.0 & 0.3 & 99.2 \\
\hline
\end{tabular}

TABELA IV: Matriz de confusão ao usar a técnica de chãoteto (seção IV-A) e 10 atributos GLCM.

\section{Análise de resultados}

A matriz de confusão da tabela I em comparação com a tabela II apresenta uma redução pouco significativa de acerto, quando consideradas as classes Normal vs Falhas (seis classes restantes), mas uma redução significativa de custo computacional devido à retirada de atributos [15]. O mesmo ocorre nas tabelas III e IV.

Devido a este comportamento nas duas técnicas observadas, todas as proximas análises apresentadas neste trabalho utilizarão somente os 10 atributos sugeridos em [15], devido a seu custo computacional reduzido.

\section{AVALIAÇÃO DA REDUÇÃO NO TAMANHO DA AMOSTRA}

Como mostrado na figura 2, essa etapa ocorre antes da extração de características e, por isso, a exploração é feita para os dois modos de extração. A partir desses resultados será escolhida a configuração a ser aplicada na plataforma embarcada.

\section{A. Usando redução na taxa de amostragem}

Nesse experimento são aplicados os métodos descritos na seção V-A para geração dos conjuntos de dados com taxa de amostragem reduzida, seguindo o fluxo da figura 2. As caracteristicas são extraídas usando as duas técnicas sob avaliação, chão-teto (seção IV-A) e SLCM (seção II). As características das amostras são entregues ao classificador, seguindo o processo de treinamento e teste já descrito na seção VI.

A tabela $\mathrm{V}$ apresenta os resultados obtidos para os dois métodos de extração. Os valores de Acurácia e de F1-Score do classificador SVM são a média de 50 rodadas de execução do experimento. São usados somente os 10 atributos GLCM sugeridos em [15], devido a seu custo computacional reduzido.

\begin{tabular}{|l|l|l|l|l|}
\hline \multirow{2}{*}{ Experimento } & SLCM & \multicolumn{2}{|l|}{ Chão-teto } \\
\cline { 2 - 5 } & Acurácia & F1-Score & Acurácia & F1-Score \\
\hline Original & 90.88 & 90.83 & 91.04 & 90.99 \\
\hline M1 & 67.80 & 67.79 & 67.92 & 67.87 \\
\hline M2 & 56.82 & 56.34 & 56.65 & 56.15 \\
\hline M3 & 62.42 & 62.05 & 62.48 & 62.06 \\
\hline M4 & 71.97 & 71.83 & 72.12 & 71.94 \\
\hline M5 & 69.78 & 69.52 & 69.33 & 69.10 \\
\hline M6 & 57.95 & 57.27 & 58.34 & 57.60 \\
\hline
\end{tabular}

TABELA V: Resultados do experimento variando o tamanho da amostra pela redução da taxa de amostragem.

\section{B. Usando redução do tempo de amostragem}

Nesse experimento são aplicados os métodos descritos na seção V-B para geração dos conjuntos de dados com corte binário do tamanho do sinal, seguindo o fluxo da figura 2 . As caracteristicas são extraídas usando as duas técnicas sob avaliação, chão-teto (seção IV-A) e SLCM (seção II). As características das amostras são entregues ao classificador, seguindo o processo de treinamento e teste já descrito na seção VI.

A tabela VI apresenta os resultados obtidos para os dois métodos de extração. Os valores de Acurácia e de F1-Score do classificador SVM são a média de 50 rodadas de execução do experimento. São usados somente os 10 atributos GLCM sugeridos em [15], devido a seu custo computacional reduzido.

\begin{tabular}{|l|l|l|l|l|}
\hline \multirow{2}{*}{ Experimento } & \multicolumn{2}{|l|}{ SLCM } & \multicolumn{2}{l|}{ Chão-teto } \\
\cline { 2 - 5 } & Acurácia & F1-Score & Acurácia & F1-Score \\
\hline Original & 90.88 & 90.83 & 91.04 & 90.99 \\
\hline M1 & 89.14 & 89.05 & 89.40 & 89.31 \\
\hline M2 & 88.94 & 88.79 & 88.38 & 88.26 \\
\hline M3 & 87.10 & 86.98 & 85.45 & 85.33 \\
\hline M4 & 84.71 & 84.54 & 82.21 & 82.09 \\
\hline M5 & 78.35 & 78.25 & 76.43 & 76.38 \\
\hline M6 & 72.82 & 72.77 & 69.47 & 69.44 \\
\hline
\end{tabular}

TABELA VI: Resultados do experimento variando o tamanho da amostra pela técnica de corte binário.

\section{Análise de resultados}

Observando os resultados da tabela $\mathrm{V}$ nota-se perdas significativas no desempenho do classificador ao reduzir a taxa de amostragem. Portanto, essa abordagem de redução não será utilizada na versão embarcada.

Já o resultado mostrado na tabela VI, indica que o classificador tolera bem as reduções no tamanho da amostra, sugerindo que essa técnica pode ser usada sem perda significativa 
da sua capacidade de generalização. Os resultados do $M 3$ (amostras de 12500 pontos) apresentam acurácia acima de $85 \%$ e serão usados para avaliação na plataforma embarcada.

\section{AVALIAÇÃO NA PLATAFORMA EMBARCADA}

A plataforma escolhida consiste em uma STM32F429ZI [16]. O trabalho [15] demonstra que ao utilizar o extrator de caractéristicas GLCM sua matriz de co-ocorrência pode ser reduzida sem perda de generalidade do classificador. Nesse momento do trabalho é utilizada uma matriz de co-ocorrência com 7 bits, reduzindo o consumo de memória desta matriz em $75 \%$.

As taxas de acerto encontradas em estação de trabalho foram de $88,05 \%$ para o método SLCM e $85,98 \%$ para o método de chão e teto, resultados similares ao encontrado na Tabela VI que utiliza uma matriz de co-ocorrência de 8bits.

Na plataforma embarcada em conjunto com a técnica proposta foi analisada a técnica de geração de atributos de chão e teto. Devido a característica do SLCM de recebimento do sinal e montagem da matriz de co-ocorrência simultaneamente, foi proposta uma implementação chamada de SLCM-Otimizado. Nesta implementação somente os valores anterior e atual do sinal são armazenada na memória de dados, juntamente com um contador, quando o número maximo de pontos é atingido o processo de captura do sinal e montagem da matriz de coocorrência é encerrado. A viabilidade dessa abordagem decorre do SLCM se basear somente na vizinhança de dados para a sua computação inicial, dispensando a presença de todo o sinal na memória. Esta otimização faz um melhor uso da memória de dados e será utilizado para comparação nas seções a seguir.

\section{A. Análise de uso de memória}

A análise da memória de dados, foi divida em 5 blocos a fim de evidenciar o ganho em cada tecnica:

1) Sinal: Consiste na memória utilizada para armazenamento do sinal. O sinal é armazenado em inteiro de 8 bits, através da digitalização para entrada do calculo da matriz de co-ocorrencia.

2) Imagem: Espaço reservado para alocação da imagem gerada. Os algoritmos clássicos, como o chão e teto, transformam o sinal em imagem, mas o SLCM não.

3) Matriz de co-ocorrência: Espaço reservado para a matriz de co-ocorrência. Esta matriz quadrada de tamanho 128 , é de tamanho fixo para todos os métodos comparados. O valor máximo assumido pelos elementos dessa matriz está associado à resolução da imagem. Considerando uma amostra de $n \times m$ pixels, esse valor está limitado a $n \times(m-1)$. Nessa implementação esse valor seria 12432 , que requer uma inteiro de 16 bits para sua representação.

4) Atributos: Espaço para armazenamento dos 10 atributos extraídos da matriz de co-ocorrência. Armazenados em variáveis de ponto flutuante 32 bits.

5) Outras variáveis: Outras variavéis para controle de fluxo de código e outras atividades não relevantes para a comparação dos métodos.

\begin{tabular}{|c|c|c|c|}
\hline Memória RAM (BYTES) & chão-teto & SLCM & SLCM-Otimizado \\
\hline Sinal & 12500 & 12500 & 2 \\
\hline Imagem & 12544 & 0 & 0 \\
\hline Matriz de co-ocorrência & 65536 & 65536 & 65536 \\
\hline Atributos & 40 & 40 & 40 \\
\hline Outras variáveis & 2152 & 2156 & 2158 \\
\hline TOTAL & 92772 & 80232 & 67736 \\
\hline
\end{tabular}

TABELA VII: Resultados de uso de memória de dados(RAM) na plataforma embarcada.

Os resultados para a memória de dados podem ser vistos na tabela VII. Os métodos chão e teto e SLCM armazenam o sinal integralmente na memória, após a digitalização feita pelo conversor analógico-digital (ADC). Entretanto, o chão e teto cria uma imagem a partir do sinal, enquanto o método de SLCM converte o sinal diretamente na matriz de co-ocorrência, não utilizando memória para esse armazenamento intermediário. Utilizando o SLCM-otimizado o armazenamento do sinal é reduzido somente a dois valores, o anterior e o atual. A comparação é realizada a cada novo dado vindo do ADC e introduzida a contagem diretamente na matriz de co-ocorrência. As três técnicas geram matrizes de co-ocorrência do mesmo tamanho.

\begin{tabular}{|c|c|c|c|}
\hline Memória FLASH (BYTES) & chão-teto & SLCM & SLCM-Otimizado \\
\hline Funções de decisão & 50232 & 64344 & 64344 \\
\hline Vetores Suporte & 48800 & 51160 & 51160 \\
\hline Outras variáveis/Código & 23856 & 24168 & 23696 \\
\hline TOTAL & 122888 & 139672 & 139200 \\
\hline
\end{tabular}

TABELA VIII: Resultados de uso de memória de programa na plataforma embarcada.

A análise de memória de programa foi dividida em três blocos, onde a maior parte das alterações de consumo ocorrem, sendo eles:

1) Funções de decisão: Estas funções fazem parte da tomada de decisão do classificador SVM do tipo Onevs-One. Para todas as técnicas, o classificador SVM utiliza um total de 21 funções de decisão com diferentes quantidades de vetores suporte em cada, causando alteração no consumo de memória, dependendo do modelo treinado para cada caso.

2) Vetores Suporte: Estes vetores suporte compõem os hiperplanos do classificador SVM. O total de vetores suporte depende do número de vetores necessários em cada função de decisão, e a essa quantidade depende do modelo treinado.

3) Outras variáveis/Código: Outras variavéis de sistema para ativação de periféricos e suporte ao programa compilado.

No microcontrolador, a memória de programa também pode ser utilizada para todas as constantes em tempo de execução. A tabela VIII apresenta os resultados obtidos nas diferentes técnicas aplicadas. Os métodos que utilizam SLCM como base possuem o mesmo modelo importado para o classificador SVM causando um consumo muito similar de memória de programa. O modelo de classificação da técnica chao-teto utiliza ao todo 
1220 vetores suporte, contra um total de 1279 no modelo usado pelo SLCM. Isto explica o consumo superior de memória de programa.

\section{B. Análise de desempenho do processo de classificação}

A análise de desempenho em tempo de computação foi divida em 3 medições. O objetivo é evidenciar a influência da técnica de extração de características e o modelo do classificador. Sendo elas:

1) Discretização e criação da CM (matriz de coocorrência): Tempo para varredura da imagem gerada e discretização para geração da matriz de co-ocorrencia.

2) Extração de atributos: Tempo para extração de atributos através das estatísticas do GLCM.

3) Classificação SVM: Tempo para realizar a classificação dos atributos extraídos.

\begin{tabular}{|c|c|c|c|}
\hline Desempenho (ms) & chão-teto & SLCM & SLCM-Otimizado \\
\hline Criação da CM & 5,0994 & 4,9401 & 4,9399 \\
\hline Extração de atributos & 387,7249 & 389,2843 & 389,7671 \\
\hline Classificação SVM & 40,9979 & 43,2133 & 43,2138 \\
\hline TOTAL & 433,8222 & 437,4377 & 437,9208 \\
\hline
\end{tabular}

TABELA IX: Resultados de desempenho do processo de classificação na plataforma embarcada.

A Tabela IX mostra na etapa de Criação da $C M$ que o método de chão-teto apresenta um valor um pouco mais elevado quando comparado com a técnica proposta, o SLCM.

$\mathrm{Na}$ etapa de Classificação SVM a diferença de tempo é resultante da quantidade de vetores suporte entre os modelos gerados em cada técnica.

\section{CONCLUSÃO}

Este artigo propõe uma nova abordagem para utilização do GLCM em sinais no tempo. O objetivo é evitar a criação de uma imagem intermediária para representação do sinal de entrada. A abordagem é avaliada usando um conjunto de dados para detecção de falhas em motores de indução, em comparação com diferentes técnicas similares encontradas na literatura. Duas abordagens para reduzir a quantidade de recursos computacionais no processo de aquisição são discutidas.

Após a validação dos experimentos, as alterações no conjunto de dados original resultaram em uma redução de 87,5\% (100.000 para 12.500) no número de pontos de aquisição necessários para gerar um resultado de classificação. Depois de validar essas explorações de conjunto de dados em uma estação de trabalho, o classificador com melhor precisão em cada técnica foi embarcado em um microcontrolador e o tempo de execução e o uso de memória foram avaliados.

A técnica proposta não apresenta uma diferença significativa em relação a tempo de computação. Entretanto, apresenta uma redução importante no custo de memória volátil que pode chegar a 27\% (92.772 para 67.736 bytes) quando comparado a outras técnicas na literatura, sem que estas mudanças afetam a capacidade de diagnóstico do classificador.
Para trabalhos futuros, novos conjuntos de dados serão pesquisados para avaliar a técnica proposta em diferentes problemas. A avaliação da técnica proposta usando um classificador embarcado conectado a um motor real está em andamento.

\section{REFERENCES}

[1] V. N. Ghate and S. V. Dudul, "Optimal mlp neural network classifier for fault detection of three phase induction motor," Expert Systems with Applications, vol. 37, no. 4, pp. 3468-3481, 2010.

[2] S. Nandi and H. A. Toliyat, "Condition monitoring and fault diagnosis of electrical machines-a review," in Conference Record of the 1999 IEEE Industry Applications Conference. Thirty-Forth IAS Annual Meeting (Cat. No. 99CH36370), vol. 1. IEEE, 1999, pp. 197-204.

[3] K. Pandey, P. Zope, and S. Suralkar, "Review on fault diagnosis in three-phase induction motor," MEDHA-2012, Proceedings published by International Journal of Computer Applications (IJCA), 2012.

[4] S. Morsalin, K. Mahmud, H. Mohiuddin, M. R. Halim, and P. Saha, "Induction motor inter-turn fault detection using heuristic noninvasive approach by artificial neural network with levenberg marquardt algorithm," in 2014 International Conference on Informatics, Electronics \& Vision (ICIEV). IEEE, 2014, pp. 1-6.

[5] M. B. K. Bouzid and G. Champenois, "Neural network based method for the automatic detection of the stator faults of the induction motor," in 2013 International Conference on Electrical Engineering and Software Applications. IEEE, 2013, pp. 1-7.

[6] S. S. Abdeldayem and T. Bourlai, "Automatically detecting arrhythmiarelated irregular patterns using the temporal and spectro-temporal textures of ecg signals," in 2018 24th International Conference on Pattern Recognition (ICPR). IEEE, 2018, pp. 2301-2307.

[7] R. M. Haralick, K. Shanmugam, and I. H. Dinstein, "Textural features for image classification," IEEE Transactions on systems, man, and cybernetics, no. 6, pp. 610-621, 1973.

[8] L.-K. Soh and C. Tsatsoulis, "Texture analysis of sar sea ice imagery using gray level co-occurrence matrices," IEEE Transactions on geoscience and remote sensing, vol. 37, no. 2, pp. 780-795, 1999.

[9] H. Wang, X.-H. Guo, Z.-W. Jia, H.-K. Li, Z.-G. Liang, K.-C. Li, and Q. He, "Multilevel binomial logistic prediction model for malignant pulmonary nodules based on texture features of ct image," European journal of radiology, vol. 74, no. 1, pp. 124-129, 2010.

[10] P. H. F. de Sousa, N. M. M. e Nascimento, P. P. Rebouças Filho, and C. M. S. de Medeiros, "Detection and classification of faults in induction generator applied into wind turbines through a machine learning approach," in 2018 International Joint Conference on Neural Networks (IJCNN). IEEE, 2018, pp. 1-7.

[11] P. H. F. de Sousa, M. Navar de Medeiros, J. S. Almeida, P. P. Rebouças Filho, V. H. C. de Albuquerque et al., "Intelligent incipient fault detection in wind turbines based on industrial iot environment," Journal of Artificial Intelligence and Systems, vol. 1, no. 1, pp. 1-19, 2019.

[12] R. Islam, J. Uddin, and J.-M. Kim, "Texture analysis based feature extraction using gabor filter and svd for reliable fault diagnosis of an induction motor," International Journal of Information Technology and Management, vol. 17, no. 1-2, pp. 20-32, 2018.

[13] W.-C. Jang, M. Kang, and J.-M. Kim, "Fault classification of an induction motor using texture features of vibration signals," in Frontier and Innovation in Future Computing and Communications. Springer, 2014, pp. 177-183.

[14] C. Cortes and V. Vapnik, "Support-vector networks," Machine learning, vol. 20, no. 3, pp. 273-297, 1995.

[15] E. T. Silva Jr, F. Sampaio, L. C. da Silva, D. S. Medeiros, and G. P. Correia, "A method for embedding a computer vision application into a wearable device," Microprocessors and Microsystems, p. 103086, 2020.

[16] S. Datasheet, "Stmicroelectronics, 2018." 